\title{
El desarrollo de la competencia de liderazgo en adolescentes en la ciudad de Tijuana
}

\author{
FELIPE DE JESÚS FERNÁNDEZ-BARBA ${ }^{a}$, \\ YOLANDA HEREDIA-ESCORZA ${ }^{b}$
}

pp. 37-52

\section{HISTORIA DEL ARTÍCULO}

¿CÓMO CITAR?:

Fernández-Barba, F. \& HerediaEscorza, Y. (2018). El desarrollo de la competencia de liderazgo en adolescentes en la ciudad de Tijuana. Perspectiva Empresarial, 5(2), 37-52. http://dx.doi. org/10.16967/rpe.v5n2a3

RECIBIDO: 5 de diciembre de 2017 APROBADO: 20 de mayo de 2018

CORRESPONDENCIA:

Yolanda Heredia Escorza. Tecnológico de Monterrey. Av. Eugenio Garza Sada No. 2501, Col Tecnológico, CP 64849. Edificio CEDES, semisótano 1, Oficina 10034, Monterrey, Nuevo León, México.

PALABRAS CLAVE adolescencia. liderazgo.

a Candidato a Doctor en educación, Proyecto Salesiano Tijuana A.C., México. Correo electrónico: feliferba15@hotmail.com

b Doctora en política social, Tecnológico de Monterrey, México. Correo electrónico: yheredia@itesm.mx 


\section{¿CÓMO CITO EL ARTÍCULO? HOW TO CITE THIS PAPER?}

CHICAGO:

Fernández-Barba, Felipe y Heredia-Escorza, Yolanda. 2018. "El desarrollo de la competencia de liderazgo en adolescentes en la ciudad de Tijuana". Perspectiva Empresarial 5(2): 37-52. http://dx.doi.org/10.16967/ rpe.v5n2a3

MLA:

Fernández-Barba, Felipe y Heredia-Escorza, Yolanda. “El desarrollo de la competencia de liderazgo en adolescentes en la ciudad de Tijuana". Perspectiva Empresarial 5.2 (2018): 37-52. Digital. http://dx.doi. org/10.16967/rpe.v5n2a3

\section{Developing Leadership Skills in Teenagers in Tijuana}

ABSTRACT This research focused on describing leadership development in five components in teenagers in Tijuana, based on the transformational leadership theory, with a mixed qualitative-quantitative approach in an exploratory design by consecutive stages. For the first stage, a leadership baseline was prepared with a sample of 165 teenagers. In the second stage, eleven teenagers participated in a program designed to train leaders, five of which were selected for the sample. The main results showed that adolescents prone to leadership know themselves and how to state their strengths and weaknesses and are positive and optimistic. Generally, they consider themselves to be full of energy and curiosity. There are signs of a transition from a conventional to a postconventional moral development stage. Their values are religious, ecological and affective. They move from an intuitive to a sensory cognitive strategy, which demonstrates the passage from concrete to abstract thinking. There are signs of greater awareness of the consequences of their actions and of the ability of self-management. They have general knowledge of what a leader is, perceive themselves as leaders and know what skill they should develop.

KEYWORDS adolescence, leadership.

\section{O desenvolvimento da competência de liderança em adolescentes na cidade de Tijuana}

RESUMO A presente pesquisa está centrada em descrever o desenvolvimento da liderança em cinco componentes em adolescentes, na cidade de Tijuana, com base na teoria da liderança transformacional, com um enfoque misto QUANTITATIVo e QUALITATIVO em um planejamento exploratório por etapas consecutivas. Para a primeira etapa foi elaborada uma linha base da liderança com uma mostra de 165 adolescentes. Na segunda etapa participaram 11 adolescentes em um programa orientado à formação de líderes, dos quais cinco foram selecionados para a mostra. Os principais resultados revelaram que os adolescentes propensos à liderança têm um conhecimento sobre si e, além disso, sabem enunciar as suas fortalezas e debilidades, são positivos e otimistas. Em geral, eles se consideram pessoas cheias de energia e curiosidade. Expressam signos de uma transição da etapa de desenvolvimento moral convencional ao pós-convencional. Seus valores são os religiosos, ecológicos e afetivos. Eles transitam de uma estratégia cognitiva de tipo intuitiva a uma sensorial, o que demonstra a transição do pensamento concreto ao abstrato. Há signos de uma maior conscientização sobre as consequências dos seus atos, assim como da capacidade de autogestão. Além disso, também têm um conhecimento geral sobre o que é um líder, percebem-se como líderes e sabem que características devem desenvolver.

PALAVRAS CHAVE adolescência, liderança. 


\section{Introducción}

Uno de los temas que ha recibido la atención tanto de académicos como de la sociedad en general es el liderazgo, pues se considera una actividad fundamental para el logro de los objetivos de todo grupo humano. De ahí que mucha investigación se ha realizado en torno a las cualidades, las acciones y la formación que se requiere para la generación de líderes, desde hace más de 70 años.

La literatura de la que se dispone da cuenta de seis teorías que explican el liderazgo: la teoría del gran hombre, la teoría de los rasgos, el comportamiento del líder y los estilos de liderazgo; los modelos contingentes, el liderazgo situacional y los modelos normativos, así como el modelo transaccional y transformacional (Hoffman y Frost, 2006; House y Beatz, 1976; Katz, 1974; Kouzes y Posner, 2003; McCall y Lombardo, 1988; Stogdill, 1974; Whetten y Cameron, 1991).

La gran mayoría de la investigación se enfocó en personas adultas, en ambientes empresariales y militares, por tanto, relativamente poco se ha investigado en relación con el camino evolutivo que el desarrollo de esta competencia sigue desde los niños hasta los adultos. Poca atención se ha dado a qué tipo de actividades educativas son necesarias para el desarrollo del liderazgo, así como a identificar las ventajas que los escenarios educativos ejercen para este mismo fin.

Al desglosar el liderazgo, este se conforma de hasta 10 componentes, cuyo dominio requiere del desarrollo y la práctica que, por lo general, le toma a la persona varias décadas de ejercicio. El principal objeto de estudio de la presente investigación es el desarrollo del liderazgo en los adolescentes, los cuales tienen la inmensa necesidad de pertenecer a grupos de referencia para desarrollarse psicológica y socialmente a fin de madurar. Para esto requieren de la conformación de una identidad personal que se fragua con la aceptación de los pares o iguales, con la orientación de figuras significativas y en actividades que les ofrecen un sentimiento de logro, además de darse el tiempo suficiente para reflexionar sobre una escala de valores, creencias y actitudes bajo una identidad propia, pero en concordancia con una cultura (Erickson, 1968, citado por Berck, 2001).

México es un país de adolescentes y jóvenes. De acuerdo con el Censo de Población del 2010, en este país habitan 112,3 millones de personas, de las cuales 36,2 millones se encuentran en un rango de edad entre 12 y 29 años, lo que representa un 32\% de la población total. Del grupo de adolescentes y jóvenes, el 50,8\% de ellos son mujeres, y el 49,2\% varones (Instituto Nacional de Estadística y Geografía-INEGI, 2016).

Sobre su condición económica, el 32,1\% de los adolescentes y jóvenes entre 14 y 29 años solo trabaja; el 11,2\% trabaja y estudia; y el 6,7\% busca trabajo o emprender un negocio.

El 12,9\% de los adolescentes y los jóvenes participan en alguna asociación o grupo; de ellos, en una de tipo deportivo el $71 \%$ de los varones y el $46 \%$ de las mujeres; de tipo estudiantil, el $28 \%$ los varones y el $33 \%$ de las mujeres; y de tipo religioso, el $16 \%$ de los varones y el $37 \%$ de las mujeres (Instituto Nacional de la Juventud-INJUVE, 2016).

Para el estado de Baja California, la estadística censal señala que la población total del estado fue de 3,1 millones de habitantes, de los cuales 891142 se encuentran en un rango de edad entre los 10 y los 24 años, lo que representa el 28\% de la población total, cifra muy cercana a la media nacional y cuyo perfil educativo y laboral es semejante (INEGI, 2016).

Se considera que México, actualmente, atraviesa por una ventana poblacional que se denomina "bono demográfico", esto es, un periodo de tiempo en el cual aumenta la cantidad de personas productivas en relación con las que aún son dependientes (menores de 15 años); el bono demográfico tiene una duración de 20 años (INJUVE, 2016).

Si bien los demógrafos consideran esta ventana de tiempo como una ventaja para todo país, en razón a la posibilidad de generar riqueza por la relación entre la población económicamente activa y la que no, lo cierto es que para logar esa ventaja es necesario consolidar a la población actual de adolescentes y jóvenes como personas plenamente desarrolladas, económicamente activas y formados en la ciudadanía. A fin de lograrlo es necesario ofrecerles servicios públicos de buena calidad tales como el servicio educativo, el de salud y la generación de empleo, primordialmente.

Se puede y debe invertir más en educación, preparación, capacitación, salud, cultura, participación, acceso a la justicia, oportunidades de empleo formal y vivienda, entre otras, a fin de que esta población joven no solo goce plenamente de sus derechos, sino que ponga las bases para una sociedad más justa, equitativa e igualitaria en este lapso de 20 años (INJUVE, 2016). 
La ventaja de tiempo de 20 años implica la entrada al siglo XxI, en el cual las habilidades tradicionales como, por ejemplo, saber leer y escribir, o el uso del razonamiento matemático, serán aún habilidades críticas; sin embargo, por sí solas no serán suficientes para tener éxito en el mundo globalizado y competitivo al que se enfrentarán las generaciones de niños y adolescentes cuando sean adultos.

Organizaciones como, por ejemplo, la Organización de las Naciones Unidas para la Educación, la Ciencia y la Cultura (Unesco, por sus siglas en inglés), la Organización para la Cooperación y el Desarrollo Económicos-OCDE (2003; 2015), y la Partnership for 21st Century Skills (2009; 2015), entre otras, han señalado la necesidad de seleccionar y definir, desde inicios del presente siglo, las competencias que se creen serán relevantes y necesarias para preparar adecuadamente a las siguientes generaciones.

La formación de los adolescentes y los jóvenes se ha centrado, en la actualidad, en el paradigma socioconstructivista, además de la educación basada en competencias.

Se define competencias como un complejo integrado por conocimientos, habilidades, actitudes, valores y atributos personales que se manifiestan en comportamientos que permiten a la persona enfrentar situaciones complejas (Lozano y Herrera, 2012). Una competencia requiere de tiempo para su adquisición, desarrollo y pleno dominio. Establecer el nivel de desempeño que una persona ha alcanzado en una competencia específica, en un momento determinado de su formación, es la forma de medirlas y evaluarlas. En el propósito de alcanzar el pleno dominio de una competencia determinada se requiere que la persona se encuentre en un ambiente educativo amplio y estructurado, de forma tal que se fomente un sentimiento de comunidad en el que los miembros de la comunidad socialicen códigos mediados por las acciones de los expertos. Esto es, las competencias maduran en términos de práctica con la estrecha mediación de los expertos y con el tiempo.

El desarrollo de las competencias para la vida, en general, y la de liderazgo en particular, en los niños, adolescentes y jóvenes, es y ha sido una preocupación constante debido a las exigencias de la vida social. Los grupos sociales van desde una familia hasta una nación, sin importar su tamaño o su complejidad, y existen porque cada colectivo tiene objetivos comunes. Se puede decir que la función del liderazgo existe cuando dos o más personas quieren alcanzar objetivos comunes.

Como ya se mencionó, se ha llevado a cabo desde hace más de 70 años-una gran cantidad de investigación en torno a las cualidades, las acciones y la formación que se requiere para la generación de líderes (Hoffman y Frost, 2006; House, y Beatz, 1976; Katz, 1974; Kouzes y Posner, 2003; McCall y Lombardo, 1988; Stogdill, 1974; Whetten y Cameron, 1991). La literatura de la que se dispone en la actualidad da cuenta de seis teorías que explican el liderazgo. Sin embargo, conviene precisar que la gran mayoría de estas investigaciones se enfocaron en identificar el liderazgo en personas adultas, en ambientes empresariales y militares, por lo que se ha investigado relativamente poco en relación con el camino evolutivo que el desarrollo del liderazgo sigue desde los niños hasta los adultos. Se ha concedido poca atención al desarrollo de este en adolescentes, al tipo de actividades educativas que son necesarias para el desarrollo del liderazgo, así como al hecho de identificar las ventajas que los escenarios educativos ejercen para este fin.

Resulta complicado evaluar un constructo que, además de tener variadas definiciones (como lo es el liderazgo), tiene una gran variedad de componentes asociados. Algunas definiciones incluyen la medición de comportamientos que facilitan la acción colectiva y la consecución de objetivos comunes, y califican a los líderes efectivos por su desempeño y efectividad, mientras que otros miden la conducta observada o la participación en ciertas actividades. Esta dificultad para medir el liderazgo se centra, a veces, en habilidades personales como, por ejemplo, la popularidad o la cantidad de grupos en la que participa, tanto en los adultos como en los adolescentes.

El liderazgo ha sido un tema estudiado por dos disciplinas. En la psicología se dio en dos vertientes. En un primer momento se intentó identificar los rasgos de personalidad que poseían aquellas personas que se habían destacado como líderes (mucha investigación se dedicó a esto), hasta llegar a la conclusión de que no era posible señalar un conjunto específico de rasgos o características que todos ellos tuvieran (House y Aditya, 1997; Katz, 1974; Marquart y Dean, 1993; Whetten y Cameron, 1991; Yukl, 1970).

En otro intento de la psicología se encaminaron los esfuerzos a identificar aquellos comportamientos que los líderes tenían. Múltiples estudios llegaron a la conclusión de que el 
comportamiento debía ser ajustado al contexto del líder y a las características de las personas que tenía a su cargo (Fiedler, 1967; House, 1971; House y Mitchell, 1974).

Otra ciencia que ha estudiado de forma exhaustiva el liderazgo es la administración, ya que en el ámbito de las organizaciones, bien sean estas con o sin fines de lucro, la función del liderazgo tiene un gran peso en el cumplimiento de sus objetivos. De esta forma, actualmente se pueden identificar seis grandes teorías que señalan las características y los comportamientos tanto de los líderes como de aquellos que los siguen, todo esto en ambientes laborales tales como los empresariales y militares, con personas adultas ya formadas.

Si bien estos esfuerzos han arrojado mucha información al respecto, desde la perspectiva educativa existe muy poca investigación dedicada a identificar cómo se desarrolla el liderazgo desde la infancia o la adolescencia, y casi nada se sabe en relación con las características de los ambientes educativos que fomentan su desarrollo, ni del proceso de enseñanza y aprendizaje que se debe realizar o el tipo de estrategias y materiales didácticos adecuados para este fin, en especial en México. Ofrecer oportunidades de formación a adolescentes es una forma de contribuir tanto a su desarrollo personal como al del país, pues son estos adolescentes los que en un plazo de 10 a 15 años ejercerán el liderazgo para conducir al país. De esta problemática surgen las siguientes preguntas generales de investigación: ¿Cuál es el nivel de la competencia de liderazgo y sus componentes en adolescentes de Tijuana de la escuela secundaria cercana a los oratorios? ¿Cómo se desarrolla la competencia de liderazgo en adolescentes en el Oratorio Salesiano en Tijuana mediante la participación en un programa de formación de líderes?

De este modo, de las preguntas de investigación se derivan algunas preguntas específicas en relación con los componentes que constituyen el liderazgo:

- ¿Cómo se enriquece el autoconocimiento en los adolescentes, antes, durante y después de participar en el programa de formación de líderes?

- ¿Cómo se desarrolla la habilidad para influir en las demás personas, antes, durante y después de participar en el programa de formación de líderes?
- ¿Cómo se desarrolla la habilidad para anticiparse al futuro, antes, durante y después de participar en el programa de formación de líderes?

- ¿Cómo se desarrolla la habilidad para planear y organizar, antes, durante y después de participar en el programa de formación de líderes?

- ¿Cómo se desarrolla la habilidad para contribuir al desarrollo de otras personas, antes, durante y después de participar en el programa de formación de líderes?

La definición de liderazgo ha cambiado, en razón a que se ha definido de muchas maneras; a continuación, se mencionan algunas:

- Se define como el proceso de dirigir el comportamiento de las personas por una vía que les permita alcanzar algunos objetivos, esto es, dirigir se entiende como llevar a las personas a actuar de cierta manera, a seguir un curso determinado de acción (Fleisheman, 1991).

- El liderazgo es la influencia interpersonal que se ejerce en una situación dada y se lleva a cabo a través del proceso de comunicación humana con el fin de alcanzar objetivos específicos. Los elementos que caracterizan al liderazgo son cuatro: la influencia, la situación, el proceso de comunicación y los objetivos a alcanzar (Chiavenato, 2004).

- El liderazgo es la habilidad para influir en las personas de modo que alcancen los objetivos, por lo que el líder participa con otras personas, lo que lo convierte en una acción recíproca que ocurre siempre entre personas (Daft, 1994).

- De forma más sencilla es la capacidad de influir a un grupo con el objetivo de alcanzar sus metas (Robbins, 2002).

- El liderazgo se define como la capacidad de influir sobre los grupos o los individuos anticipándose al futuro y contribuyendo al desarrollo personal, profesional o comunitario (Villa y Poblete, 2007).

De estas definiciones se pueden extraer tres elementos: la capacidad de influir, la capacidad de anticiparse al futuro y la capacidad de contribuir al desarrollo de otras personas. La capacidad de influencia se relaciona con la forma en que los líderes comunican su visión a los seguidores, para 
los cuales la opinión y los consejos del líder son importantes y merecen escucharlos y seguirlos. La segunda característica es la capacidad de anticiparse al futuro; el líder tiene la capacidad de ver con claridad aquellas situaciones posibles en un periodo de tiempo, y esto le permite orientar la acción del grupo hacia este futuro posible y deseable. La tercera característica es la de contribuir al desarrollo de las demás personas, pues los líderes tienen una fuerte orientación hacia el servicio, así como la actitud proactiva de planear y ejecutar proyectos que beneficien a los individuos y a su comunidad, y no solo a ellos mismos.

Las personas pueden desarrollar el liderazgo si reciben la orientación necesaria para ello, y si viven experiencias adecuadas que les ayuden a lograrlo, sin perder de vista que existen personas que tienen de manera natural mayores fortalezas y cualidades, y para quienes lograr el dominio de la competencia es más sencillo (Villa y Poblete, 2007).

Se presentan, a continuación, las teorías, que se consideran clásicas.

- La teoría del gran hombre. Se considera el carisma como una cualidad de la personalidad individual, por la cual se considera a alguien como excepcional con relación a las personas ordinarias, y, por tanto, se la trata como a un dotado de poderes o cualidades sobrenaturales o excepcionales. Se centró en el estudio de las grandes figuras de la humanidad, por lo general aquéllas de carácter religioso, político o militar (House y Beatz, 1976; Hoffman y Frost, 2006; Kouzes y Posner, 2003; McCall y Lombardo, 1988; Stogdill, 1974).

- La teoría de los rasgos. Intentó describir cuáles eran las principales características y habilidades que distinguían a los grandes líderes (House y Aditya, 1997; Katz, 1974; Marquart y Dean, 1993; Whetten y Cameron, 1991; Yukl, 1970).

- La teoría conductista. Presenta dos líneas; por un lado, la investigación sobre las características del trabajo directivo, es decir, qué actividades, funciones y responsabilidades asumen los directivos y cómo distribuyen su tiempo; por otro, los estudios sobre las conductas de los directivos eficaces (Blake y McCanse, 1991; Blake y Mouton 1978; Lewin, Lippit y White, 1939; Likert, 1961; Short y Greer, 2002).
- La teoría de la contingencia. Aspira a describir un estilo de liderazgo adecuado y contingente a factores como, por ejemplo, las relaciones del líder con los miembros, el tipo de miembros o seguidores, el clima o cultura organizativa y otros factores ambientales. Su postulado se refería a que los líderes que conquistan el éxito son aquellos que pueden adaptar su comportamiento para conciliar las exigencias de su propia y única situación. Esta teoría se basa en la cantidad de conducción (orientación hacia la tarea) y la cantidad de apoyo socio-emocional (orientación hacia las relaciones) que un líder debe aportar dada la situación, así como el "nivel de madurez" del grupo supervisado (Fiedler, 1967; House, 1971; House y Mitchell, 1974).

- La teoría del liderazgo situacional. El líder debería elegir los patrones de liderazgo más adecuados para cada una de las situaciones en que se encuentre. En este sentido, el liderazgo es un fenómeno circunstancial que tiene tres fuentes: 1. La fuerza del líder, sus características personales, sus valores, la confianza que tiene en los subordinados, las inclinaciones sobre como dirigir, su capacidad de delegar y la tolerancia a la ambigüedad; 2. La fuerza de los subordinados, sus características, su necesidad de autonomía, el deseo de asumir responsabilidades, la tolerancia a la incertidumbre, la comprensión del problema y las competencias desarrolladas; y 3. La fuerza de la situación, las condiciones en que se ejerce el liderazgo, las características de la organización, su estructura y cultura, el ambiente de trabajo y el clima organizacional (Hersey y Blanchard; 1977; Tannenbaum y Schmidt, 1978).

- La Teoría del liderazgo transaccional y transformacional. Al estudiar el liderazgo político Burns (1978) identificó dos tipos de liderazgo: el transaccional que implica solo un intercambio entre líderes y seguidores, y el transformacional, el cual se fundamenta en el cambio de valores y creencias, así como en la satisfacción de las necesidades de los seguidores. Los líderes transaccionales intercambian premios por esfuerzos o promesas de premios por un buen desempeño, reconocen el éxito, buscan e identifican las desviaciones de las reglas y de los patrones para aplicar acciones correctivas, intervienen solo cuando no se alcanzan 
los parámetros, dan libertad total al renunciar a su responsabilidad y evitan tomar decisiones, de forma que si algo sale mal, ellos no tiene culpa alguna. Los líderes transformacionales proporcionan una visión y una misión, infunden orgullo y merecen el respeto y la confianza, comunican grandes expectativas, utilizan símbolos para enfocar los esfuerzos, expresan propósitos importantes con métodos simples, promueven la inteligencia, la racionalidad y una cuidadosa solución de problemas. Asimismo, brindan atención personal, esto es, tratan a cada persona de forma individual, la asesoran y la impulsan.

Para esta investigación se ha seleccionado un conjunto acotado de componentes que conforman el liderazgo, los cuales se consideraron los más apropiados para adolescentes. Se extraen de los modelos de Whetten y Cameron (1991), Ricketts y Rudd (2002), y de Villa y Poblete (2007), así como de los principios de la teoría del líder transformacional (Bass, 1985). Los componentes seleccionados son: autoconocimiento, influir en los demás, anticiparse al futuro, planeación y organización, y contribuir al desarrollo de los demás.

\section{Método}

El enfoque de la presente investigación fue de tipo mixto, la cual, dadas sus intenciones, es de tipo exploratorio con un diseño por etapas consecutivas CUAN, CUAL, también denominado "diseño secuencial explicativo" (Creswell y Plano, 2007). La elección de un diseño mixto se fundamentó en la poca existencia de información previa sobre el desarrollo de liderazgo en adolescentes mexicanos que pudiera ofrecer antecedentes para describirlo, establecer un nivel de dominio y explicarlo.

Las dos fases que componen el estudio se definieron de la siguiente manera: la fase cuantitativa (CUAN) tuvo como objetivo establecer una línea base sobre el nivel de desarrollo de liderazgo en una muestra no probabilística de adolescentes provenientes de las escuela secundaria cercana al Oratorio, por medio de la aplicación de una batería de instrumentos adecuados a los elementos que componen el liderazgo. Esta línea base se tomó como un parámetro, pues no existe información sobre el nivel de dominio del liderazgo en el adolescente.
Se diseñó la propuesta pedagógica de programa de formación de líderes bajo un enfoque de competencia. Se utilizó el formato modular para cada uno de los componentes del modelo de liderazgo propuesto por los cinco componentes. Se colocó el programa de formación de líderes en una plataforma electrónica o LMS (Schoology).

En la fase cualitativa de la investigación (CUAL), la que brindó la mayor riqueza de la información, se llevó a cabo una muestra por conveniencia compuesta por cinco miembros del grupo de adolescentes que tomaron el programa. Se recopilaron las evidencias que cada participante colocó en la plataforma y se evaluaron con las rúbricas específicas. La fase cuantitativa de la investigación tuvo como escenario la Escuela Secundaria N. ${ }^{\circ} 6$ de Tijuana, ya que es la única cercana al Oratorio. La fase cualitativa tuvo como escenario el Oratorio Salesiano, una institución con un significado especial para todos aquellos que forman parte de la familia salesiana fundada por Juan Bosco (2003).

En la fase cuantitativa se calculó y seleccionó una muestra no probabilística para una población finita y con tamaño conocida, esto es, la población de alumnos de la Escuela Secundaria N. ${ }^{\circ}$ 6 en el turno matutino, la cual estaba compuesta por 550 alumnos. Se calculó la muestra con la fórmula adecuada, y dio como resultado 165 sujetos. Para la fase cualitativa acudieron 11 participantes al programa de formación de líderes. Este grupo se autoseleccionó, ya que fueron los adolescentes quienes, de forma voluntaria, participaron. De este grupo de adolescentes el análisis se centró en cinco de ellos: los que demostraron un mayor potencial de liderazgo.

Por tanto, en la fase cuantitativa, los sujetos fueron 165 adolescentes con una edad que oscila entre los 13 y los 15 años, y se consideró tanto a hombres como a mujeres que aceptaron resolver los instrumentos de medición en su escuela.

En la fase cualitativa, los participantes fueron 11 adolescentes con las mismas características de los anteriores (edad y sexo), quienes realizaron el programa de formación de liderazgo; de ellos se seleccionó a cinco para el estudio.

Los instrumentos y las técnicas utilizadas para recopilar información se presentan la tabla 1.

La estrategia de análisis de datos consistió en la calificación de los test, de acuerdo con los lineamientos que aparecen en sus manuales. Con esta información se registró en la base de datos y se procedió a realizar el análisis estadístico descriptivo; 
TABLA 1. Instrumentos y técnicas utilizadas

\begin{tabular}{|c|c|c|c|}
\hline $\begin{array}{l}\text { FASE DE LA } \\
\text { INVESTIGACIÓN }\end{array}$ & $\begin{array}{l}\text { ELEMENTOS DE LA } \\
\text { COMPETENCIA }\end{array}$ & INSTRUMENTO A UTILIZAR & INFORMACIÓN QUE RECOPILA \\
\hline \multirow{9}{*}{ Cuantitativa } & Liderazgo & $\begin{array}{l}\text { Multifactor Leadership } \\
\text { Questionnarie (MLQ), versión } \\
\text { corta. Versión en español. }\end{array}$ & $\begin{array}{l}\text { Este test mide nueve factores que se } \\
\text { consideran parte del liderazgo transaccional y } \\
\text { transformacional. }\end{array}$ \\
\hline & Autoconocimiento & & \\
\hline & $\begin{array}{l}\text { Autoconocimiento } \\
\text { Escala de valores }\end{array}$ & $\begin{array}{l}\text { Test de valores de Casares } \\
\text { (1995). }\end{array}$ & $\begin{array}{l}\text { El test establece la jerarquía de valores } \\
\text { personales de los adolescentes. }\end{array}$ \\
\hline & $\begin{array}{l}\text { Autoconocimiento. } \\
\text { Estilo cognitivo }\end{array}$ & $\begin{array}{l}\text { Instrumento de estilo } \\
\text { cogntivo (The cogntivie style } \\
\text { instrument), Rotter (1966). }\end{array}$ & $\begin{array}{l}\text { Establece la forma en que la persona obtiene y } \\
\text { evalúa la información del medio. }\end{array}$ \\
\hline & $\begin{array}{l}\text { Autoconocimiento } \\
\text { Locus de control }\end{array}$ & $\begin{array}{l}\text { Escala de Locus de control } \\
\text { (Locus of Control Scale) de } \\
\text { Julian Rotter. }\end{array}$ & $\begin{array}{l}\text { Determina la forma en que la persona ejerce el } \\
\text { control sobre su conducta. }\end{array}$ \\
\hline & $\begin{array}{l}\text { Autoconocimiento } \\
\text { Inteligencia emocional }\end{array}$ & $\begin{array}{l}\text { Cuestionario de inteligencia } \\
\text { emocional para alumnos de } \\
\text { seis a } 18 \text { años, } \\
\text { de Prieto et al. (2011). }\end{array}$ & $\begin{array}{l}\text { Establece el nivel de los componentes de la IE. } \\
\text { Mide cuatro subescalas. }\end{array}$ \\
\hline & $\begin{array}{l}\text { Capacidad de influir en } \\
\text { otras personas }\end{array}$ & $\begin{array}{l}\text { Rúbrica para evaluar ensayos } \\
\text { persuasivos. }\end{array}$ & $\begin{array}{l}\text { Capacidad de redactar un ensayo de tipo } \\
\text { persuasivo. }\end{array}$ \\
\hline & $\begin{array}{l}\text { Planeación y } \\
\text { organización de } \\
\text { anticiparse al futuro }\end{array}$ & Problema de la NASA. & $\begin{array}{l}\text { Capacidad de encontrar la solución a un } \\
\text { problema determinado. }\end{array}$ \\
\hline & $\begin{array}{l}\text { Contribuir al desarrollo } \\
\text { de los demás }\end{array}$ & $\begin{array}{l}\text { Cuestionario de inteligencia } \\
\text { emocional. Para alumnos de } \\
\text { seis a } 18 \text { años, de Prieto et } \\
\text { al. (2011). }\end{array}$ & $\begin{array}{l}\text { Puntaje de las subescalas de modo intra e } \\
\text { interpersonal. }\end{array}$ \\
\hline \multirow{5}{*}{ Cualitativa } & Historia de vida & $\begin{array}{l}\text { Cédulas de entrevista } \\
\text { semiestructurada. }\end{array}$ & $\begin{array}{l}\text { Narrativa para reunir información que } \\
\text { permita establecer eventos de la vida de los } \\
\text { participantes (infancia, relaciones con los } \\
\text { padres, vida escolar, asistencia al Oratorio, } \\
\text { etc.). }\end{array}$ \\
\hline & $\begin{array}{l}\text { Vivencia sobre el } \\
\text { programa }\end{array}$ & $\begin{array}{l}\text { Cédula de entrevista } \\
\text { semiestructurada. }\end{array}$ & $\begin{array}{l}\text { Narrativa de los pensamientos y sentimientos } \\
\text { con relación al desarrollo de los componentes } \\
\text { del liderazgo. }\end{array}$ \\
\hline & $\begin{array}{l}\text { Incidentes en la sesión } \\
\text { del programa }\end{array}$ & Nota de campo. & $\begin{array}{l}\text { Redacción de la forma en que se realizó la } \\
\text { sesión del programa. }\end{array}$ \\
\hline & $\begin{array}{l}\text { Descripción de las } \\
\text { vivencias del instructor } \\
\text { e investigador }\end{array}$ & $\begin{array}{l}\text { Diario del instructor e } \\
\text { investigador. }\end{array}$ & $\begin{array}{l}\text { Redacción de los pensamientos, los } \\
\text { sentimientos del instructor del programa y del } \\
\text { investigador. }\end{array}$ \\
\hline & $\begin{array}{l}\text { Análisis de las } \\
\text { evidencias de } \\
\text { desempeño de cada } \\
\text { participante }\end{array}$ & $\begin{array}{l}\text { Rubricas de evaluación de } \\
\text { las evidencias. }\end{array}$ & $\begin{array}{l}\text { Establecer la forma en que cada uno de los } \\
\text { elementos de liderazgo evolucionó en cada } \\
\text { participante }\end{array}$ \\
\hline
\end{tabular}

Fuente: elaboración propia

finalmente, con estos datos se construye la línea base. El mismo procedimiento de análisis para los resultados obtenidos por la muestra cualitativa en sus aplicaciones previas y posteriores a su participación en el programa de formación de líderes se aplicará antes y después del programa. La fase cualitativa siguió el modelo de tratamiento de datos cualitativos enunciado por Wolcott (1994), citado por Strauss y Corbin (2002), el cual consta de tres componentes: la descripción, el análisis y la interpretación. 


\section{Resultados}

A fin de integrar los elementos que pueden ser de utilidad a los adolescentes en el propósito de enriquecer su conocimiento de sí mismos se aplicaron diversas medidas. En las siguientes tablas se muestra los resultados de la línea base, así como los que obtuvieron los participantes en el programa de formación en cada uno de los test psicométricos.

Se puede observar un orden diferente entre la escala promedio de la línea base y las que se obtuvieron por el grupo que realizó el programa de formación de líderes y la muestra seleccionada de este grupo en dos versiones: la previa al programa y la posterior.

Se aprecian algunos cambios y coincidencias: en dos de las escalas los valores religiosos pasaron del último lugar en la línea base, al primero en el grupo de adolescentes que decidieron tomar el programa de formación de líderes, seguidos por los valores ecológicos en tres de las escalas, lo cual confirma que hay un aprecio por el medio

TABLA 2. Resultados comparados en la escala de valores

\begin{tabular}{|c|c|c|c|c|}
\hline VALORES & $\begin{array}{l}\text { LIINEA BASE } \\
\mathbf{N}=165\end{array}$ & $\begin{array}{l}\text { GRUPO DEL PROGRAMA DE } \\
\text { FORMACIÓN DE LÍDERES } \\
\mathrm{N}=11\end{array}$ & $\begin{array}{l}\text { MUESTRA CUALITATIVA } \\
\text { INICIAL } \\
\mathbf{N}=5\end{array}$ & $\begin{array}{l}\text { MUESTRA CUALITATIVA } \\
\text { POSTERIOR } \\
\mathbf{N}=5\end{array}$ \\
\hline Morales & 37 & 44 & 39 & 30 \\
\hline Ecológicos & 36 & 32 & 41 & 48 \\
\hline Afectivos & 35 & 38 & 36 & 42 \\
\hline Sociales & 35 & 32 & 38 & 38 \\
\hline Individuales & 33 & 44 & 40 & 30 \\
\hline Intelectuales & 33 & 48 & 34 & 38 \\
\hline Corporales & 30 & 32 & 26 & 36 \\
\hline Instrumentales & 29 & 38 & 21 & 10 \\
\hline Estéticos & 25 & 44 & 27 & 40 \\
\hline Religiosos & 18 & 48 & 34 & 48 \\
\hline
\end{tabular}

Fuente: elaboración propia.

TABLA 3. Resultados comparados del estilo cognitivo

\begin{tabular}{|c|c|c|c|c|}
\hline \multicolumn{3}{|c|}{ PROCESO ADQUISITIVO DE INFORMACIÓN } & \multicolumn{2}{|c|}{ EVALUACIÓN RESPECTO A LA INFORMACIÓN RECIBIDA } \\
\hline & Intuitiva & Sensorial & Basada en pensamiento & Basada en sentimientos \\
\hline $\begin{array}{l}\text { Línea base } \\
\mathrm{N}=165\end{array}$ & 2,17 & 1,78 & 3,65 & 2,25 \\
\hline $\begin{array}{l}\text { Grupo del programa de formac } \\
\text { de líderes } \\
\mathrm{N}=11\end{array}$ & 2,3 & 1,63 & 3,9 & 2,18 \\
\hline $\begin{array}{l}\text { Muestra cualitativa. Inicial } \\
\mathrm{N}=5\end{array}$ & 2,6 & 1,4 & 4 & 2 \\
\hline $\begin{array}{l}\text { Muestra cualitativa. Posterior } \\
\mathrm{N}=5\end{array}$ & 2,2 & 2 & 5 & 1 \\
\hline
\end{tabular}

Fuente: elaboración propia.

TABLA 4. Resultados comparados de locus de control

\begin{tabular}{lllll}
\hline $\begin{array}{l}\text { LOCUS DE } \\
\text { CONTROL }\end{array}$ & $\begin{array}{l}\text { LÍNEA BASE } \\
\mathbf{N}=165\end{array}$ & $\begin{array}{l}\text { GRUPO DEL PROGRAMA DE } \\
\text { FORMACIÓN DE LIDERES } \\
\mathrm{N}=11\end{array}$ & $\begin{array}{l}\text { MUESTRA CUALITATIVA } \\
\text { INICIAL } \\
\mathbf{N}=5\end{array}$ & $\begin{array}{l}\text { MUESTRA CUALITATIVA } \\
\text { POSTERIOR } \\
\mathbf{N}=5\end{array}$ \\
\hline Interno & 13,62 & 12,20 & 12,20 & $\mathbf{1 6 , 0 0}$ \\
\hline Externo & $\mathbf{1 4 , 6 2}$ & $\mathbf{1 6 , 3 0}$ & $\mathbf{1 6 , 2 0}$ & 12,80 \\
\hline
\end{tabular}

Fuente: elaboración propia. 
y la naturaleza — que es un valor universal一, tal como se mencionó en la línea base.

En las mediciones de los diversos grupos se confirmó que la estrategia intuitiva para la obtención de información es la que caracteriza a los adolescentes. En cuanto a la estrategia para la evaluación de la información, de todas las mediciones realizadas la estrategia basada en razonamiento es la que se privilegia.

Tres de las mediciones sitúan al locus de control externo por encima del interno, únicamente la medición posterior al programa de la muestra cualitativa, en la que aparece el locus de control interno. Se puede especular que el hecho de someter a los adolescentes a una comunidad en la que se habló y reflexionó sobre valores, así como sobre la responsabilidad personal, y se vieron ejemplos concretos de líderes adolescentes, haya influido para este cambio en la percepción sobre las consecuencias de la acción personal.
La escala intrapersonal es la que obtiene el mayor puntaje en el grupo de adolescentes que participaron en el programa desde antes de hacerlo y de nuevo después de haberlo hecho. Esta parte de la inteligencia emocional se encuentra relacionada con la capacidad de percibir y expresar las emociones propias y con la capacidad para la intromisión. Los adolescentes que participaron en el curso de formación de líderes en la medición posterior al curso mejoran en el puntaje de la escala interpersonal, la cual es la base para establecer relaciones positivas con los demás y la base de la orientación hacia el servicio.

La capacidad para influir en las demás personas medidas con la redacción de un mensaje de tipo persuasivo obtiene los puntajes que se presentan en la tabla 6.

La redacción de textos persuasivos obtuvo su mejor puntaje en la muestra cualitativa al final del programa de formación de líderes, después de

TABLA 5. Resultados comparados de inteligencia emocional

\begin{tabular}{lcccc}
\hline ESCALAS & $\begin{array}{l}\text { LíNEA BASE } \\
\mathbf{N}=165\end{array}$ & $\begin{array}{l}\text { GRUPO DEL PROGRAMA DE } \\
\text { FORMACIÓN DE LÍDERES } \\
\mathbf{N}=11\end{array}$ & $\begin{array}{l}\text { MUESTRA CUALITATIVA } \\
\text { INICIAL } \\
\mathbf{N}=5\end{array}$ & $\begin{array}{l}\text { MUESTRA CUALITATIVA } \\
\text { POSTERIOR } \\
\mathbf{N}=5\end{array}$ \\
\hline Interpersonal & 2,11 & 2,31 & 2,32 & 2,54 \\
\hline Intrapersonal & $\mathbf{2 , 6 7}$ & 2,82 & $\mathbf{2 , 9 3}$ & $\mathbf{3 , 2 4}$ \\
\hline $\begin{array}{l}\text { Escala de manejo del } \\
\text { estrés }\end{array}$ & 2,12 & 1,84 & 1,94 & 2,15 \\
\hline $\begin{array}{l}\text { Escala de } \\
\text { adaptabilidad }\end{array}$ & 2,55 & 2,84 & 3,04 & 3 \\
\hline
\end{tabular}

Fuente: elaboración propia.

TABLA 6. Resultados comparados de la redacción de textos persuasivos

\begin{tabular}{|c|c|c|c|c|}
\hline CRITERIOS & $\begin{array}{l}\text { LÍNEA BASE } \\
\mathrm{N}=165\end{array}$ & $\begin{array}{l}\text { GRUPO DEL PROGRAMA DE } \\
\text { FORMACIÓN DE LIIDERES } \\
\mathbf{N}=11\end{array}$ & $\begin{array}{l}\text { MUESTRA CUALITATIVA } \\
\text { INICIAL } \\
\mathbf{N}=5\end{array}$ & $\begin{array}{l}\text { MUESTRA CUALITATIVA } \\
\text { POSTERIOR } \\
\mathrm{N}=5\end{array}$ \\
\hline Claridad y coherencia & 2,12 & 2,27 & 2,4 & 3 \\
\hline Síntesis de contenidos & 2,01 & 2,27 & 2,4 & 2,8 \\
\hline Uso del lenguaje & 1,93 & 2,18 & 2,4 & 2,8 \\
\hline $\begin{array}{l}\text { Capacidad de } \\
\text { convencimiento }\end{array}$ & 2,03 & 2,09 & 2,4 & 3 \\
\hline Puntaje global & 2,25 & 2,25 & 2,4 & 2,9 \\
\hline
\end{tabular}

Fuente: elaboración propia. 
dedicar tiempo a concientizar sobre la importancia de la comunicación.

TABLA 7. Resultados comparados de los aciertos de un desastre en la luna

\begin{tabular}{|c|c|}
\hline ACIERTOS & \\
\hline Línea base. $\mathrm{N}=165$ & 1,3 \\
\hline $\begin{array}{l}\text { Grupo del programa de formación de líderes. } \\
\mathrm{N}=11\end{array}$ & 1,22 \\
\hline Muestra cualitativa Inicial. $\mathrm{N}=5$ & 1 \\
\hline Muestra cualitativa Posterior. $\mathrm{N}=5$ & 2,4 \\
\hline
\end{tabular}

El ejercicio sobre el desastre en la luna obtuvo un mejor puntaje después del programa, pero aún está lejos de ser un buen resultado.

El estilo predominante en todos los casos es el transformativo, aunque la diferencia con el estilo transaccional es de tan solo un punto o menos en las diversas mediciones.

Los resultados del análisis cualitativo se derivaron de las notas de campo de las sesiones, de las evidencias colocadas por los participantes en la plataforma, de las entrevistas y del diario del investigador. En la tabla 9 se presentan de acuerdo con el componente de liderazgo.

TABLA 8. Resultados comparados del estilo de liderazgo

\begin{tabular}{lccccc}
\hline ESTILO & $\begin{array}{l}\text { LÍNEA BASE } \\
\mathbf{N}=165\end{array}$ & $\begin{array}{l}\text { GRUPO DEL PROGRAMA DE } \\
\text { FORMACIÓN DE LÍDERES } \\
\mathbf{N}=11\end{array}$ & $\begin{array}{l}\text { MUESTRA CUALITATIVA } \\
\text { INICIAL } \\
\mathbf{N}=5\end{array}$ & $\begin{array}{l}\text { MUESTRA CUALITATIVA } \\
\text { POSTERIOR } \\
\mathbf{N}=5\end{array}$ \\
\hline Estilo transformacional & 9,26 & 10,8 & 12,8 & 12 \\
\hline Estilo transaccional & 8,30 & & 9,9 & 10,5 & 11,7 \\
\hline Estilo laissez faire & 6,37 & & 6,5 & 6,6 & 6,6 \\
\hline
\end{tabular}

Fuente: elaboración propia.

TABLA 9. Resultado cualitativo de cada componente

- Los adolescentes de la muestra tienen un conocimiento sobre sí mismos que han acumulado a lo largo de su vida.

- Son capaces de enunciar sus fortalezas y de reconocer sus debilidades.

- Se describen en términos positivos, con una mirada equilibrada sobre ellos mismos.

- La información de los resultados de los test y la reflexión sobre ellos incrementó lo que ya sabían y enriqueció este conocimiento. Es necesario además de entregar los resultados de los test, dar un tiempo para que cada uno los comprenda e integre aquella información a su conocimiento de sí mismo.

AUTOCONOCIMIENTO - En general, se consideran personas optimistas, llenas de energía y curiosidad.

- Se notan las diferencias individuales (timidez, extroversión, reflexividad), y se fue creando la atmósfera de comunidad en la que se comparten temas e intereses, lo que generó un sentimiento de confianza y seguridad.

- Los cinco adolescentes elegidos son reconocidos por sus compañeros por ser carismáticos, tienden a escucharlos y tomar sus comentarios en cuenta, y son los que más participan en las sesiones grupales.

- Tienen un conocimiento general sobre lo qué es un líder y las características que debe desarrollar, pero no se conciben a sí mismos como líderes al inicio del curso.

- Los adolescentes comprenden la intencionalidad de un mensaje para convencer a otros.

- Tienen argumentos para sus posturas, pero aún no las presentan con claridad o de forma convincente.

- Pueden perder la concentración necesaria para sostener un argumento por medio de una conversación o por escrito.

- Se notan diferencias en la capacidad de cada uno para enfrentar a una audiencia, aunque sea un público compuesto de por compañeros.

INFLUIR EN LOS DEMÁS

- Tienen un

- manejo adecuado para su edad del lenguaje tanto escrito como oral, lo cual se ha desarrollado en casa y en la escuela.

- Pueden desarrollar aún más las cuatro habilidades lingüísticas con ejercicios lúdicos pero dirigidos a esta finalidad.

- Identifican que este aspecto es uno de los más importantes para los líderes. 
Continuación TABLA 9.

- Se encuentran relatos muy detallados de la historia personal, pero no discriminan entre un relato más fluido u ordenado de eventos importantes.

- Se describen historias de familias integradas y con relaciones positivas y amorosas.

- Se relatan historias académicas positivas con maestros y compañeros.

ANTICIPARSE AL FUTURO - Se descubren gustos e intereses que han realizado desde niños.

- Son capaces de identificar las necesidades de una organización y de identificar qué información les es relevante.

- Los participantes colectan la información, pero no separan lo relevante o importante y se quedan con el cúmulo de información colectada.

- Se descubren intenciones muy claras en relación con aquellas actividades o profesiones que quieren desempeñar en el futuro.

- Reconocen el apoyo que reciben y los compromisos que deben asumir

- Tienen cimientos sobre cómo elaborar un plan de trabajo o presupuesto que se presentó en diversos formatos.

- Fue necesario buscar información más especializada para fundamentar mejor los presupuestos.

- Presentan capacidad de análisis de la información, pero aún debe desarrollarse.

- Son capaces de crear y usar formatos diferentes para fines diferentes.

- Para casi todos fue la primera experiencia de servicio en la que participaban y había expectación y un poco de ansiedad.

- En esta actividad, que requiere de un contacto más o menos cercano con muchas personas de diversa índole (algunos sucios, o con mal olor, enojados o turbulentos), se da un fuerte torrente de emociones entre quien los sirve, pero fueron muy bien manejadas por los adolescentes.

CONTRIBUIR AL DESARROLLO DE LOS DEMÁS
- Control emocional.

- Sentimiento de gusto y satisfacción por tener la oportunidad de dar algo a las demás personas.

- El descubrimiento de las necesidades de otros y las que uno mismo tiene satisfechas gracias a su familia.

Fuente: elaboración propia.

\section{Conclusiones}

Los adolescentes proclives al liderazgo que se encuentran en el rango de edad de entre 13 y 15 años de la muestra, presentan un conocimiento acumulado a lo largo de su vida sobre sí mismos, y son capaces de hacer una descripción de su aspecto físico y psicológico bastante acertada. Conocen sus fortalezas y limitaciones y pueden expresarlas con claridad. Están respaldados por familias integradas con relaciones amorosas y positivas, o al menos así las perciben los propios adolescentes. Reconocen los factores que los pueden beneficiar o dañar. Tiene un proyecto de vida en mente basado en un interés personal, aun cuando no lo han fundamentado con hechos.

Expresan una escala de valores personales que no coinciden con las de otros adolescentes, pero precisamente este hecho los coloca en un peldaño más avanzado en el desarrollo moral con convicción propia, lo que significa el paso de una moral convencional a una posconvencional y personal. A pesar de tener la necesidad de aceptación de sus pares, eligen a aquellos que son más afines a su gustos e intereses, así como a su nivel de desarrollo cognitivo y emocional, y dan muestras de una preocupación con base en valores más universales. Al terminar el programa lograron una mayor conciencia de que sus acciones tienen consecuencias, y de que ellos tienen el control sobre sus acciones como parte de la competencia de liderazgo que se basa en el autoconocimiento y en la capacidad de comandar sus acciones de forma coherente.

Sus habilidades cognitivas dan muestra de un pensamiento abstracto que depende menos de los sentidos y de las formas concretas de apreciar y evaluar la realidad, pero aún centrados en su propio criterio. Su afectividad se expresa de forma abierta, son más bien alegres y llenos de energía con una mirada hacia su interior, reconocen sus emociones y pueden expresarlas, y dan muestras de la capacidad de empatía.

$\mathrm{Al}$ ofrecérseles herramientas para incrementar el conocimiento de sí mismos, escuchan, toman para sí y procuran integrar los resultados a su conocimiento de sí mismos de forma consciente; pueden estar o no de acuerdo con los resultados, pero hacen este esfuerzo de integración de nueva información. 
Son capaces de expresarse tanto en forma oral como escrita, con una intención comunicativa. Si bien logran estructurar un discurso en el que manifiestan una postura personal, aún pueden mejorar en el uso de lenguaje, así como en lograr una mayor concentración en sus mensajes y mejorar en el dominio de las habilidades lingüísticas. No obstante, para su edad y escolaridad, presentan un buen desempeño en esa área. Identifican las habilidades de los líderes y cuando se les muestran ejemplos concretos sobre las funciones de los líderes, o sobre la capacidad de los jóvenes para cambiar el mundo, se impresionan y se identifican con estos modelos.

Cuando se les presentan escenarios reales pueden establecer una línea de tiempo, reconocer el pasado y prever un futuro. Tal como lo pueden hacer con su proyecto de vida, lo pueden establecer cuando se les sitúa en el papel de líderes. No cuentan con la información suficiente para emprender acciones, pero saben dónde encontrarla; su limitación se establece en relación con la selección y el análisis de dicha información, al separar lo importante de lo superfluo. Son capaces de seguir instrucciones y de accionar con herramientas tecnológicas. En general, sostienen la atención y terminan tareas de mediana complejidad. Pueden diseñar y utilizar formatos diversos.

Asimismo, están en capacidad de trabajar en colaboración con otros, se organizan de forma espontánea y logran combinar fortalezas individuales para lograr metas comunes. Lograron crear una comunidad y un fuerte sentimiento de pertenencia a este grupo; en ella se compartió conocimiento y se apoyaron para desarrollar habilidades.

Poseen una inclinación hacia el servicio a las demás personas; si bien es una oportunidad que no se presenta usualmente en su medio, una vez que lo han experimentado lo disfrutan y descubren que les agrada.

Desarrollan a su nivel los componentes de liderazgo de forma gradual y en la medida de su nivel de desarrollo personal.

En relación con el estilo de liderazgo, se encuentra una tendencia hacia el transformacional. De las cinco dimensiones de este modelo, se observó lo siguiente:

- La dimensión carisma y la capacidad de inspiración se pueden observar en los adolescentes elegidos, pues son los más abiertos y extrovertidos y sus compañeros los escuchan y toman sus comentarios en cuenta. No parece una postura estudiada sino algo que surge naturalmente en ellos.

- En la dimensión sobre la capacidad de comunicar una visión de los líderes, encontramos que los adolescentes de la muestra han desarrollado las habilidades lingüísticas de forma adecuada para su edad y colocan la intencionalidad en su comunicación, pero aún falta desarrollar con mayor amplitud la argumentación y la concentración; también de forma espontánea los escuchan sus pares, lo cual estimula esta dimensión de liderazgo.

- La tercera dimensión que versa sobre la capacidad del lider de dar un seguimiento y acompañamiento cercano a sus seguidores se relaciona con el componente del servicio a los demás; en el grupo de adolescentes que tomaron el programa de formación se presentó, por primera vez, la oportunidad de realizar un servicio de manera organizada y se descubrieron como capaces de hacerlo, con el control emocional adecuado y el sentimiento de satisfacción personal que esto generó. Durante las sesiones fue posible observar la colaboración y el apoyo de unos a otros de forma espontánea, lo cual son también semillas de esta tercera dimensión.

- Finalmente, la cuarta dimensión del modelo sobre la estimulación intelectual, la cual el líder debe de forma constante brindar a sus seguidores para su desarrollo, se encuentra también relacionada en la investigación con adolescentes con la forma en que estos se organizan tanto cognitiva como emocionalmente cuando se enfrentan a escenarios reales en los cuales deben resolver retos.

\section{Referencias}

Blake, R. y McCanse, A. (1991). Leadership dilemmas-grid solutions. Houston, TX: Gulf. Recuperado de https://www.amazon.com/Leadership-Dilemmas-Grid\%C2\%AE-Solutions-Organization / dp/0872014886

Blake, R. y Mouton, J. (1978). The managerial grid. Houston: Gulf.

Bass, B. M. (1985). Leadership and performance beyond expectations. Nueva York: Free Press.

Berck, L. (2001). Desarrollo del niño y del adolescente ( $4^{\underline{a}}$ ed.). Madrid: Prentice Hall.

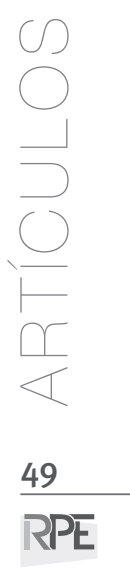


Bosco, J. (2003). El amor supera al reglamento: práctica y teoría educativa de Don Bosco. Madrid: ccs.

Burns, J. M. (1978). Leadership. Nueva York: Harper and Row.

Casares, P. (1995, septiembre-diciembre). Test de valores: un instrumento para la evaluación. Revista Española de Pedagogía, 53(202), 513-537. Recuperado de http://www.jstor.org/stable/23764771

Creswell, J. W. y Plano-Clark, V. L. (2007) Designing and conducting mixed methods research. Thousand Oaks, CA: Sage Publications.

Chiavenato, I. (2004). Comportamiento organizacional: la dinámica del éxito en las organizaciones, México: CDM. Thompson Editores.

Daft, R. L. (1994). Management (8a ed.). Fort Worth Texas: The Dryden Press.

Erikson, E. (2009). Infancia y sociedad. México, D. F.: Horme Paidós.

Fleisheman, E. A. (1991). Taxonomic effort in the description of leadership behaavior. Leadership Quarterly, 2(4), 245-289.

Fiedler, F. (1967). A theory of leadership effectiveness. Nueva York: McGraw-Hill.

Hersey, P. y Blanchard, K. (1977). Management of organizational behavior. Englewood Cliffs, CA, EUA: Prentice- Hall.

Hoffman, B. y Frost, B. (2006). Multiple intelligences of transformational leaders: an empirical examination. International Journal of Manpower, 27(1), 37-51.

Horn, L. (2011). Leadership skill development: the perceptions of connecticut 4-H adolescent 4-H leaders in the connecticut 4-H youth development program. University Of Connecticut. Recuperado de http:// gradworks.proquest.com/34/68/3468063.html

House, R. J. (1971). A path goal theory of leader effectiveness, Administrative Science Quarterly, 16, 321-329.

House, R. J. y Aditya, R. N. The Social scientific study of leadership Quo Vadis? Journal of Management, 23(3), 409-474.

House, R. J. y Beatz, J. L. (1976). Theory of charismatic leadership. En J. G. Hunt y L. Larson (Ed.), Leadership: the cutting edge (pp. 189-207). Carbondale: Southern Illinois University.

House, R. J. y Mitchell, T. R. (1974, otoño). Path-goal theory of leadership, Contemporary Business, 3, 81-98.

Instituto Nacional de Estadística y Geografía-INEGI. (2016). Población, hogares y vivienda. Inegi.org. $m x$. Recuperado de http://www3.inegi.org.mx/ sistemas/temas/default.aspx?s=est\&c=17484

Instituto Nacional de la Juventud-INJUVE. (2016). Encuesta Nacional del Jóvenes 2010. Imjuventud.gob. $m x$. Recuperado de http://www.imjuventud.gob. $\mathrm{mx} /$ pagina.php?pag_id=137
Katz, R. (1974, septiembre). Skills of an effective administrator. Harvard Business Review, 90101. Recuperado de https://hbr.org/1974/09/ skills-of-an-effective-administrator

Kouzes, J. y Posner, B. Z. (2003). Leadership practices inventory ( $3^{\mathrm{a}}$ ed.). E. E. U. U.: Pieffer.

Lewin, K., Lippit, R. y White, R. K. (1939). Patterns of aggressive behavior in experimentally created social climates, Journal of Social Psychology, 10, 271-299.

Likert, R. (1961). New patterns of management. Nueva York: McGraw-Hill.

Lozano, A. y Herrera, A. (2012). Diseño de programas educativos basados en competencias. Monterrey, $\mathrm{N}$. L.: Editorial Digital Tecnológico de Monterrey.

McCall, M. y Lombardo, M. (1988). Lessons of experience: how successful executives develop on the job. Nueva York: The Free Press.

Marquart, M. J. y Dean, W. E (1993, mayo). HRD Competencies for a shrinking world. Training and development, 62-64.

Organización para la Cooperación y Desarrollo Económico (2003). Definición y selección de competencias (DeSeCo). Resumen ejecutivo. Recuperado de http://www.deseco.admin.ch/bfs/deseco/en/ index/03/02.parsys.78532.downloadList.94248. DownloadFile.tmp/2005.dscexecutivesummary. sp.pdf

Organización para la Cooperación y el Desarrollo Económico (2015). La definición y selección de competencias clave. Resumen ejecutivo. Recuperado de http://www.deseco.admin.ch/bfs/deseco/en/ index/03/02.parsys.78532.downloadList.94248. DownloadFile.tmp/2005.dscexecutivesummary. sp.pdf

Partnership for 21st Century Skills. (2009). Framework for 21st Century Learning. Recuperado de http:// www.p21.org/about-us/p21-framework

Partnership for 21st Century Skills. (2015). P21 Framework definitions. Recuperado de 2015 http:// www.p21.org/storage/documents/docs/P21_Framework_Definitions_New_Logo_2015.pdf.

Prieto, M. D., Ferrandiz, C, Sánchez, C., Bermejo, R., Ferrando, M., Hernández, D. y Serna, B. (2011). Cuestionario de inteligencia emocional para alumnos de 6-18 años, profesores y padres. $R e$ vista Electrónica Interuniversitaria de Formación del Profesorado, 38(14,3). Recuperado de http:// www.aufop.com/aufop/uploaded_files/revistas/132818816010.pdf

Ricketts, J. C. y Rudd, R. D. (2002). A comprehensive leadership education model to train, teach, and develop leadership in youth. Journal of Career and Technical Education, 19(1), Recuperado de http:// scholar.lib.vt.edu/ejournals/JCTE/v19n1/ricketts. html 
Robbins, S. P. (2002). Comportamiento organizacional. México, D. F.: Prentice Hall.

Rotter, J. (1966). Generalized expectancies for internal versus external control of reinforcement. Psychological Monographs: General and Applied, 80(1), 1-28.

Strauss, A. y Corbin, J. (2002). Bases de la investigación cualitativa. Técnicas y procedimientos para desarrollar la teoría fundamentada. Colombia: Editorial Universidad de Antioquia.

Short, P. M. y Greer, J. T. (2002). Leadership in empowered schools: themes from innovative efforts, $\mathrm{NJ}$ : Merrill Prentice Hall.

Stogdill, R. M. (1974). Handbook of leadership: a survey of the literature. Nueva York: Free Press.

Tannenbaum, A. S. y Schmitt, W. H. (1958, marzo-abril). How to choose a leadership pattern. Harvard Business Review, 36, 95-101.
Unesco. (2013b). Technology, broadband and education advancing the education for all agenda. The Broadband Commission. United Nations Educational, Scientific and Cultural Organization (Unesco), International Telecommunication Union (ITU). Recuperado de http://www.broadbandcommission.org/work/working-groups/education/ BD_bbcomm-education_2013.pdf

Villa, A. y Poblete, M. (2007). Aprendizaje basado en competencias. Una propuesta para la evaluación de las competencias genéricas. Madrid: Mensajero.

Whetten, D. A. y Cameron, K. S. (1991). Developing management skills. Nueva York: Harper Collins.

Yulk, G. A. (2012). Leadership in organizations. NJ, E. E. U. U.: Prentice Hall. 
